\title{
Characterization of alpha-fetoprotein effects on dendritic cell and its function as effector immune response activator
}

This article was published in the following Dove Press journal: Journal of Hepatocellular Carcinoma

\section{Jeremiah Suryatenggara ${ }^{1,2}$ Heri Wibowo ${ }^{2}$ Wahyuni Lukita Atmodjo 3 George Mathew ${ }^{1,3}$}

'Mochtar Riady Institute for Nanotechnology and Medical Science Group, Pelita Harapan University, Tangerang, ${ }^{2}$ Biomedical Science Graduate Programme, Faculty of Medicine, University of Indonesia, Jakarta, ${ }^{3}$ Faculty of Medicine, Pelita Harapan University, Tangerang, Indonesia
Correspondence: Jeremiah Suryatenggara Mochtar Riady Institute for Nanotechnology and Medical Science Group, University of Pelita Harapan, Jalan Boulevard Jenderal Sudirman 1688, Lippo Karawaci, Tangerang,

Tel +62 2I $542 \mid 0123$

Fax +62 2154210110

Email jsurya@mrinstitute.org

\begin{abstract}
Tumor antigen alpha-fetoprotein (AFP) can promote immune tolerance toward tumor cells by inducing regulatory functions of the immune system. The purpose of this study was to characterize the effects of AFP on dendritic cells (DC) in their antitumor immune response stimulation and subsequent immune tolerance toward tumor cells. Monocytes were cultured in medium with GM-CSF and IL-4 and incubated for 6 days to generate immature DC (imDC). AFP was added into the treatment group at the beginning of the monocyte-derived DC culture. Mature DC (mDC) were generated by an addition of lipopolysaccharide (LPS) into the culture and incubation for another 48 hours. We observed that the addition of AFP in early DC culture was able to decrease the binding of LPS onto imDC surface, which lowered the strength of stimulation and consequently the maturity of DC. As expected, the expression of $\mathrm{mDC}$ surface markers, which are known to be crucial in effector cell proliferation and activation such as HLADR, CD40, CD80, CD83, and CD86, were confirmed to be reduced on AFP-exposed DC. DC potential in stimulating proliferation of $\mathrm{CD}^{+}{ }^{+} \mathrm{T}$ cells was decreased, in line with the reduction of surface markers' expression. Additionally, an increased secretion of cytokine TGF- $\beta$ by DC was observed. In summary, AFP inhibited the effector immune responses while increasing the regulatory immune responses in DC. This might lead to tolerance toward antigens and tumor cell survival, such as in cases of hepatocellular carcinoma patients with high levels of AFP.
\end{abstract}

Keywords: alpha-fetoprotein, dendritic cells, antigen-presenting cells, hepatocellular carcinoma, lipopolysaccharide, tumor immunoescape

\section{Introduction}

Hepatocellular carcinoma (HCC) is a form of malignancy in which increased levels of serum alpha-fetoprotein (AFP) are found in most patients. ${ }^{1-3}$ Several studies have shown that the functionalities of circulating dendritic cells $(\mathrm{DC})^{4}$ and monocyte-derived DC (MDDC) $)^{5}$ were disrupted in HCC patients. Several in vitro studies have indicated that AFP disrupts the activation of various pro-inflammatory immune responses of DC, such as reduction of pro-inflammatory cytokine secretion, ${ }^{5-7}$ and lowers the expression of functional surface markers. ${ }^{7-9}$ As a consequence, immune cells that are responsible for cellular immunity against tumor, such as T cells ${ }^{5}$ and NK cells, ${ }^{7}$ become perturbed in their activation and proliferation. This abnormality in DC function is proposed as one of the tumor escape mechanisms and as one of the main obstacles in cancer immunotherapy success. ${ }^{6}$

Successful DC maturation is indicated by significant production of proinflammatory cytokines and expression of costimulatory molecules. ${ }^{10}$ The presence of 
these factors provides complete stimulation for naïve CD4 ${ }^{+}$ T-cell clonal expansion and differentiation into type $1 \mathrm{~T}$ helper (Th1) cell, which generates pro-inflammatory immune response and plays a significant role in antitumor immune response. ${ }^{11}$ Failure in DC maturation disrupts its ability to express costimulatory molecules, which are indispensable in T-cell clonal expansion, and also in its ability to secrete pro-inflammatory cytokines, which leads to the disruption of the effector-type Th1 immune response. ${ }^{8,9}$

Pardee et al found out that AFP-treated DC decreased its ability to stimulate the proliferation of $\mathrm{T}$ cells from both subsets $\mathrm{CD}^{+}$and $\mathrm{CD} 8^{+}{ }^{12}$ This finding points out that regardless of the type of immune response generated, the strength of the generated response itself would also have been compromised already by AFP exposure.

AFP is proven to polarize immune response toward regulatory type. ${ }^{13}$ Activation of this regulatory pathway downregulates the activation and proliferation of pro-inflammatory immune components. This regulatory mechanism serves to limit the effector immune responses, thus avoiding damage towards nonforeign, nonpathogenic, or nonmalignant cells and tissues. ${ }^{10}$ Exposure of DC to such tumor-derived factors such as AFP is reported to alter the characteristics and functions of DC into a more tolerant APC, which is usually known as a tolerogenic DC. ${ }^{14}$ When compared to the normal immunogenic DC, this tolerogenic DC exhibits decreased expression of stimulatory molecules, pro-inflammatory cytokines, and pro-inflammatory transcription factors. On the other hand, it increases the expression of inhibitory, endocytosis, and apoptosis receptors, while increasing anti-inflammatory and inhibitory cytokines. ${ }^{15,16}$

Within the topic of AFP effects on DC, many researches have already provided evidences starting from the prevention of NF- $\kappa \mathrm{B}$ translocation into the nucleus to decreased T-cell proliferation. We look to widen the observation scope further outside the area of currently available evidences. In this study, we specifically observed the induction of DC maturation by lipopolysaccharide (LPS), which is far upstream from the NF- $\kappa \mathrm{B}$ translocation, and also the very start of DC activation mechanism in response to the presence of foreign immunogenic antigens. In this study, we also observed DC secretion level of TGF- $\beta$, the cytokine highly involved in the differentiation of naïve $\mathrm{CD} 4^{+} \mathrm{T}$ cells into immunosuppressive regulatory $\mathrm{T}$ cells, which inherently determines the generated immune response-type downstream of $\mathrm{CD}^{+} \mathrm{T}$-cell clonal expansion.

The study aims to provide further information regarding AFP effects, including their characteristics of interference, toward regulation of DC intracellular signals and their subsequent effects on other immune system components.
Knowing that AFP has already been indicated as an immunosuppressing factor, the result may contribute in the development of DC-based immunotherapy in HCC patients, or possibly even the development of AFP as an immunosuppressant agent in human patients. Further evidence is needed to fully describe how far the immunosuppressive effects of AFP can skew the whole DC-generated immune response into the regulatory pathway.

\section{Methods}

\section{Peripheral blood mononuclear cell isolation}

Human peripheral blood was taken from donors, which consisted of three male adults in their late 20s. Donors were clinically healthy with neither genetic disorders nor congenital abnormalities. In compliance with the Declaration of Helsinki, all donors involved in this project gave their consents with regard to having their peripheral blood taken and used in this study. Ethical approval was granted by the Committee on Health Research Ethics of the Mochtar Riady Institute for Nanotechnology. Peripheral blood mononuclear cells (PBMCs) were isolated by differential centrifugation with Ficoll Paque solution (Amersham Bioscience).

\section{Preparation of MDDC}

PBMCs were incubated for 30 minutes at $37^{\circ} \mathrm{C}$ with $5 \% \mathrm{CO}_{2}$. Medium was subsequently discarded and well surface was washed twice with PBS. Adherent monocytes (Figure 1) were further cultured in dendritic cell culture medium (DCCM), which consists of RPMI 1640 (Gibco) + 10\% FBS (Gibco) + 800 U/mL GM-CSF (BD Biosciences Pharmingen) + 1,000 U/mL IL-4 (BD Biosciences Pharmingen). DC maturation from its immature state (immature DC [imDC]) into its mature state (mature DC [mDC]) was induced by the addition of bacterial cell wall antigen LPS (Sigma), which was added into the medium on the sixth day of the MDDC culture (Figure 1). In total, $500 \mathrm{ng}$ of LPS per mL DCCM was used according to the previous publication. ${ }^{12}$ The maturation process took 48 hours at $37^{\circ} \mathrm{C}$ and $5 \% \mathrm{CO}_{2}$ (Figure 1 ).

\section{AFP treatment into MDDC culture}

AFP used in this experiment was commercially available purified human cord blood AFP (Lee Biosolutions), with $\geq 99 \%$ purity (Figure S1). Any effect caused on DC in this study is strictly within the scope of cord blood AFP obtained from a normal healthy human, contrary to the more potent and authentic tumor patient-derived AFP. AFP was added into the medium at the beginning of the culture, at a concentration of $6.25 \mu \mathrm{g}$ per $\mathrm{mL}$ DCCM. This concentration was chosen 


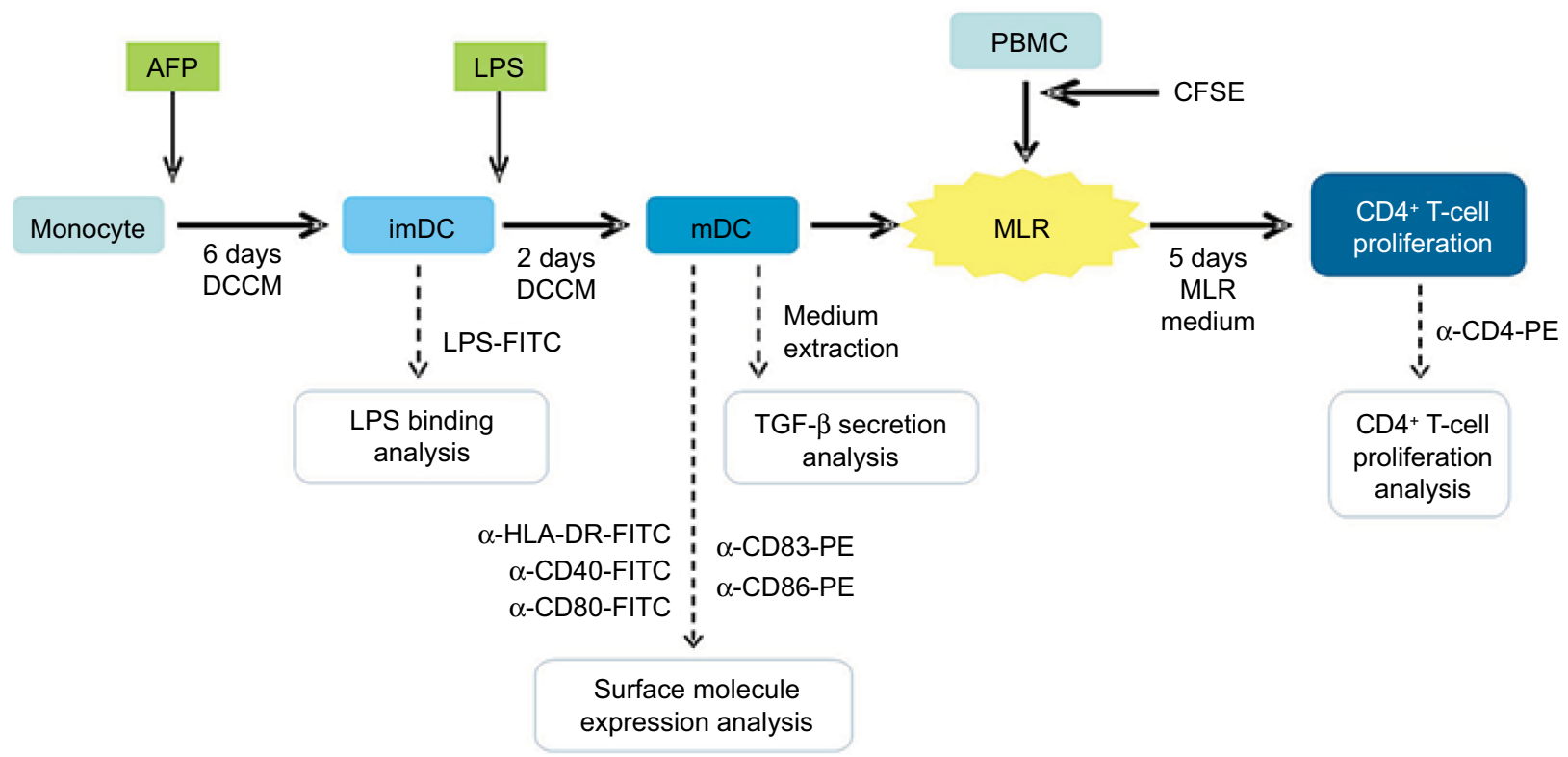

Figure I Diagram of research method.

Notes: Monocyte was cultured for 6 days to generate imDC. imDC was maturated by addition of LPS into the culture and incubation for 2 days. mDC was combined together with autologous PBMC for 5 days in a MLR culture in order to simulate the stimulation of T-cell activation and proliferation by DC. LPS binding onto DC surface was analyzed when it was still in the immature state. Expression of functional molecules and TGF- $\beta$ secretion by DC were analyzed after it has reached maturity. DC-induced CD4+ T-cell proliferation was analyzed after MLR culture with PBMC. AFP was given to the positive treatment group at the beginning of MDDC culture.

Abbreviations: imDC, immature dendritic cell; LPS, lipopolysaccharide; mDC, mature DC; PBMC, peripheral blood mononuclear cell; MLR, mixed lymphocyte reaction; MDDC, monocyte-derived DC; AFP, alpha-fetoprotein; CFSE, carboxyfluorescein succinimidyl ester; DCCM, dendritic cell culture medium.

based on a study by Um et al. ${ }^{5}$ At this concentration, AFP was able to cause an optimal reduction of DC surface molecule expression while still maintaining the culture viability $>80 \%$.

\section{Observation of AFP effect on LPS binding onto DC surface}

Immature forms of DC, both AFP-treated imDC (imDCAFP) and nontreated (imDC), were labeled with LPS-FITC (Sigma). Unlabeled untreated imDC was used as autofluorescence baseline value. Fluorescence intensity of FITC on each cell was then quantified by flow cytometry. Mean fluorescence intensity (MFI) value was obtained through $\mathrm{X}$-value mean calculation by Beckman-Coulter Epics Altra flow cytometry software (EXPO32). This observation method was applied on all three biological replicates separately on different flow cytometry runs.

Two additional experiments were performed to further investigate the AFP mechanism of action in decreasing the binding of LPS onto DC surface. First experiment was performed to confirm the binding of AFP onto DC surface after 6 days of culture and its persistence after repeated washing with PBS. Immature forms of DC, both AFP-treated (imDC-AFP) and non-treated (imDC), were washed with PBS three times before they were labeled with anti-AFP-PE (R\&D Biosciences). Unlabeled untreated imDC was used as autofluorescence baseline value. Fluorescence intensity of PE on each cell was then quantified by flow cytometry. MFI value was obtained through direct MFI calculation by Becton Dickinson Accuri C6 flow cytometry software (Csampler). This observation method was applied on three technical replicates, from one biological sample, separately on different flow cytometry runs.

The second experiment was done to observe the AFP effect on the expression of TLR4 molecules, the widely known specific receptor for LPS, on DC surface. Immature forms of DC, both AFP-treated (imDC-AFP) and non-treated (imDC), were washed with PBS three times before they were labeled with anti-TLR4-APC (R\&D Biosciences). Unlabeled untreated imDC was used as autofluorescence baseline value. Fluorescence intensity of APC on each cell was then quantified by flow cytometry. MFI value was obtained through direct MFI calculation by Becton Dickinson Accuri C6 flow cytometry software (Csampler). This observation method was applied on three technical replicates, acquired from a single biological sample, but separately on different flow cytometry runs.

\section{Detection of functional surface molecule expression on DC}

Anti-HLA-DR-FITC, anti-CD40-FITC, anti-CD83-PE, anti-CD80-FITC, and anti-CD86-PE (BD Biosciences 
Pharmingen) were used under standard surface marker labeling procedure to label their corresponding functional surface molecules on DC surface. All of the assigned DC groups were labeled with the aforementioned antibodies and read using flow cytometry (Beckman Coulter Epics Altra). Anti-CD40-FITC was co-labeled together with anti-CD83PE, while anti-CD40-FITC was co-labeled with CD83-PE. PE fluorescence spillover compensation was done on FITC channel at $6.5 \%$. FITC fluorescence spillover compensation was done on PE channel at $12.5 \%$. This observation method was applied on three technical replicates from a single donor on different flow cytometry runs.

\section{TGF- $\beta$ secretion by DC}

TGF- $\beta$ secretion by DC was observed by quantifying the TGF- $\beta$ level in the culture medium used for culturing each assigned DC group, with TGF- $\beta 1$ ELISA Kit (BD Biosciences) using standard manufacturer's protocol. This observation method was applied on all three biological replicates separately on different flow cytometry runs.

\section{Mixed lymphocyte reaction}

PBMCs to be used as mixed lymphocyte reaction (MLR) culture responder were all stained with carboxyfluorescein succinimidyl ester (CFSE; eBioscience). CFSE was added as much as $5 \mu \mathrm{M}$ into a suspension of $10 \mathrm{~mL}$ PBS $+5 \% \mathrm{FBS}$ containing 10 million PBMCs. The suspension was then incubated for 5 minutes at room temperature and washed twice with PBS and pelleted by centrifugation. Pellet was resuspended in $1 \mathrm{~mL}$ of MLR medium (90\% RPMI $+10 \%$ PBS) per $10^{6}$ PBMCs. DC was combined with PBMC in MLR culture using MLR culture medium (RPMI $+10 \%$ FBS) for 5 days in the dark at $37^{\circ} \mathrm{C}$ and $5 \% \mathrm{CO}_{2}$ (Figure 1). DC and PBMC number in each culture chamber was conditioned to achieve 1:10 stimulator-to-responder ratio. Proliferation of $\mathrm{CD} 4^{+} \mathrm{T}$ cells was observed from the fluorescence of intracellular CFSE at the end of 5 days of MLR culture, determined by flow cytometry (Beckman Coulter Epics Altra). Proliferation on $\mathrm{CD}^{+} \mathrm{T}$ cells among all PBMCs can be observed by labeling the PBMCs with antibody anti-CD4-PE prior to flow cytometry. This observation method was applied on all three biological replicates separately on different flow cytometry runs. All raw percentage values before statistical analysis were normalized according to imDC as the $100 \%$ control to see what the treatment does to the initial $100 \%$. All values were normalized according to $\mathrm{mDC}$ as the $100 \%$ control to observe how $\mathrm{mDC}$ function suffered from immunosuppression by AFP.

\section{Results}

\section{AFP decreased interaction between DC and its maturation signal LPS}

In order to observe the AFP effect on the binding of LPS onto imDC surface, we exposed DC to AFP since the first day of MDDC culture (imDC-AFP) and examined the amount of LPS bound on DC surface on day 6 by using flow cytometry analysis. Wells of DC without AFP treatment (imDC) served as control. Flow cytometry reading (Figure 2) showed a significant reduction in LPS on DC surface when compared to normal untreated imDC. Both groups are significantly different $(p=0.00533)$ (Table 1).

Additional experiment result (Figure S2) demonstrated that at the end of the monocyte-derived DC development, the AFP that was added 6 days prior was detected on the surface of imDC in a significant amount when compared to imDC groups in which AFP was not added at all ( $p=0.003642$, Table S1). This suggests that AFP is a molecule that adheres to the surface of DC, presumably to its specific receptor AFP-r. Nevertheless, the fact to prove binding between AFP and AFP-r within this experimental design needs further confirmation.

Additionally, another additional experiment result (Figure S3) demonstrated a much lower number of available TLR4 molecules in imDC group which was treated with AFP since 6 days prior, compared to the non-treated imDC group ( $p=0.004016$, Table S2). AFP-treated DC still expressed a reduced number of TLR4 molecules rather than being totally eliminated or altered by AFP treatment. This finding suggests that exposure of DC toward AFP during its differentiation from monocytes decreased the amount of expressed TLR4 on its immature-state cell surface.

\section{AFP inhibited the expression of functional surface molecules on $\mathrm{mDC}$}

Normal untreated $\mathrm{mDC}$ showed the highest expression of corresponding molecules among all groups (Figure 3). AFP treatment has no apparent effect on the difference between treated (imDC-AFP) and untreated (imDC) immature DC. On the other hand, AFP treatment consistently decreased the expression of these functional surface molecules (HLA-DR, CD40, CD83, CD80, and CD86) on mDC. AFP treatment in overall made the expressions on AFP-treated $\mathrm{mDC}$ (mDC-AFP) to fall down to a level just slightly above that of imDC (Table 2).

Statistical analysis of the aforementioned percentage values using two-tailed paired Student's $t$-test showed that 6 days of AFP treatment causes a significant decrease of all tested 


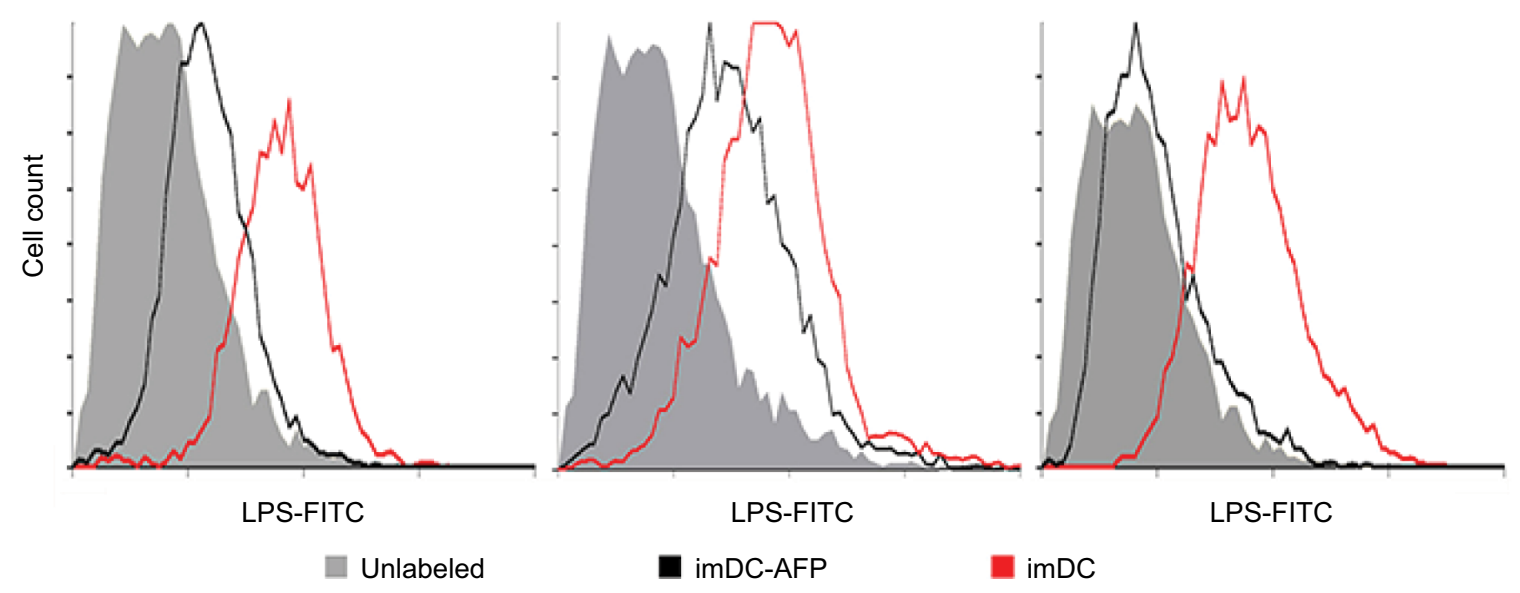

Figure 2 Flow cytometry histogram showing the fluorescence intensity of LPS-FITC on the surface of DC.

Notes: The results were obtained from three biological replicates. The fluorescence intensity at FITC light level wavelength reflected the number of LPS successfully bound onto DC surface.

Abbreviations: LPS, lipopolysaccharide; DC, dendritic cell; AFP, alpha-fetoprotein; imDC, immature DC.

Table I MFI of LPS-FITC-labeled DC

\begin{tabular}{|c|c|c|c|c|c|}
\hline \multirow[t]{2}{*}{ Group } & \multicolumn{5}{|c|}{ MFI (AU) } \\
\hline & \#I & $\# 2$ & \#3 & Average & SD \\
\hline Unlabeled & 0.30 & 0.30 & 0.30 & 0.30 & 0.00 \\
\hline imDC & 0.80 & 0.90 & 0.80 & 0.83 & 0.06 \\
\hline imDC-AFP & 0.50 & 0.60 & 0.40 & 0.50 & 0.10 \\
\hline$p$-value & \multicolumn{3}{|c|}{ imDC vs imDC-AFP } & \multicolumn{2}{|l|}{$0.00533 *$} \\
\hline
\end{tabular}

Notes: MFI values of mean fluorescence intensity from the three biological replicates are shown in the table. $p$-value between imDC and imDC-AFP groups was calculated using two-tailed paired Student's $t$-test to see the significance of AFP treatment effect on LPS binding to DC surface. ${ }^{*} p<0.05$.

Abbreviations: MFI, mean fluorescence intensity; LPS, lipopolysaccharide; DC dendritic cell; imDC, immature DC; imDC-AFP, alpha-fetoprotein-treated imDC.

functional surface molecules $(p=0.0285,0.0066,0.0021$, 0.0010 , and 0.0020 for HLA-DR, CD40, CD83, CD80, and CD86, respectively) (Table 2).

However, each molecule showed variations in the strength of DC maturation inhibition by AFP. HLA-DR, CD80, and CD86 molecule expressions were greatly inhibited in the mDC-AFP groups. There was no statistically significant difference in these three surface molecule expressions between mDC-AFP group and imDC group $(p=0.0643,0.4408$, and 0.0947 , respectively) (Table 2).

On the other hand, the effect was not as strong in the case of CD40 and CD83 molecule expressions. The percentage values between these two molecules still showed significant differences between the mDC-AFP and imDC groups ( $p=0.0176$ and 0.0375 , respectively) (Table 2). In terms of $\mathrm{CD} 40$ and CD83, and their respective functions in immune system activation, although weakened, $\mathrm{mDC}$-AFP are still more potent than immature ones.

\section{AFP increased the secretion of anti- inflammatory cytokine TGF- $\beta$}

$\mathrm{mDC}$ secreted more TGF- $\beta$ than its immature state (imDC). When treated with AFP during its differentiation from monocyte, $\mathrm{mDC}$ (mDC-AFP) secreted significantly higher level of TGF- $\beta$ than normal untreated $\mathrm{mDC}$ ( $p=0.039614$ by two-sample $t$-test). Likewise, in its immature state, AFP caused imDC (imDC-AFP) to secrete significantly higher level of TGF- $\beta$ ( $p=0.034511$ by two-sample $t$-test) (Table 3 ).

\section{AFP reduced $D C$ potential in stimulating the proliferation of $\mathrm{CD} 4^{+} \mathrm{T}$ cells}

mDC-AFP stimulated less $\mathrm{CD}^{+}{ }^{+} \mathrm{T}-$-cell proliferation than normal untreated $\mathrm{mDC}$, while the same association cannot be said between AFP-treated (imDC-AFP) and normal untreated immature form of DC (imDC) (Figure 4). There is a significant difference in $\mathrm{CD}^{+} \mathrm{T}$-cell proliferation between $\mathrm{mDC}$ and $\mathrm{mDC}-\mathrm{AFP}$ treatment groups ( $p=0.0021$ by two-sample $t$-test) (Table 4). Interestingly, similar to the numbers in the observation of functional surface molecules, it also shows that the $\mathrm{CD}^{+} \mathrm{T}$-cell proliferation percentage of $\mathrm{mDC}$-AFP group was still significantly higher than that of imDC group ( $p=0.0264$ by two-sample $t$-test), while there was not any significant effect between imDC and imDC-AFP groups ( $p=0.8999$ by two-sample $t$-test). This suggests that AFP treatment prevented DC to achieve normal $\mathrm{mDC}$ optimum potential in inducing proliferation of $\mathrm{CD} 4^{+} \mathrm{T}$ cells.

$\mathrm{DC}$ are able to show decreased $\mathrm{CD} 4^{+} \mathrm{T}$-cell proliferation when co-cultured with $\mathrm{CD} 4^{+} \mathrm{T}$ cells without the presence of AFP in the medium. While there are indeed many published 
A
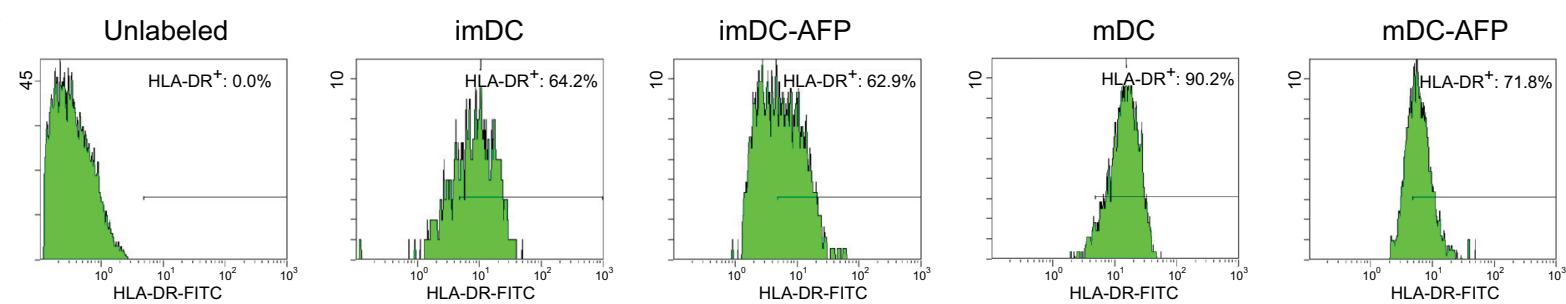

B
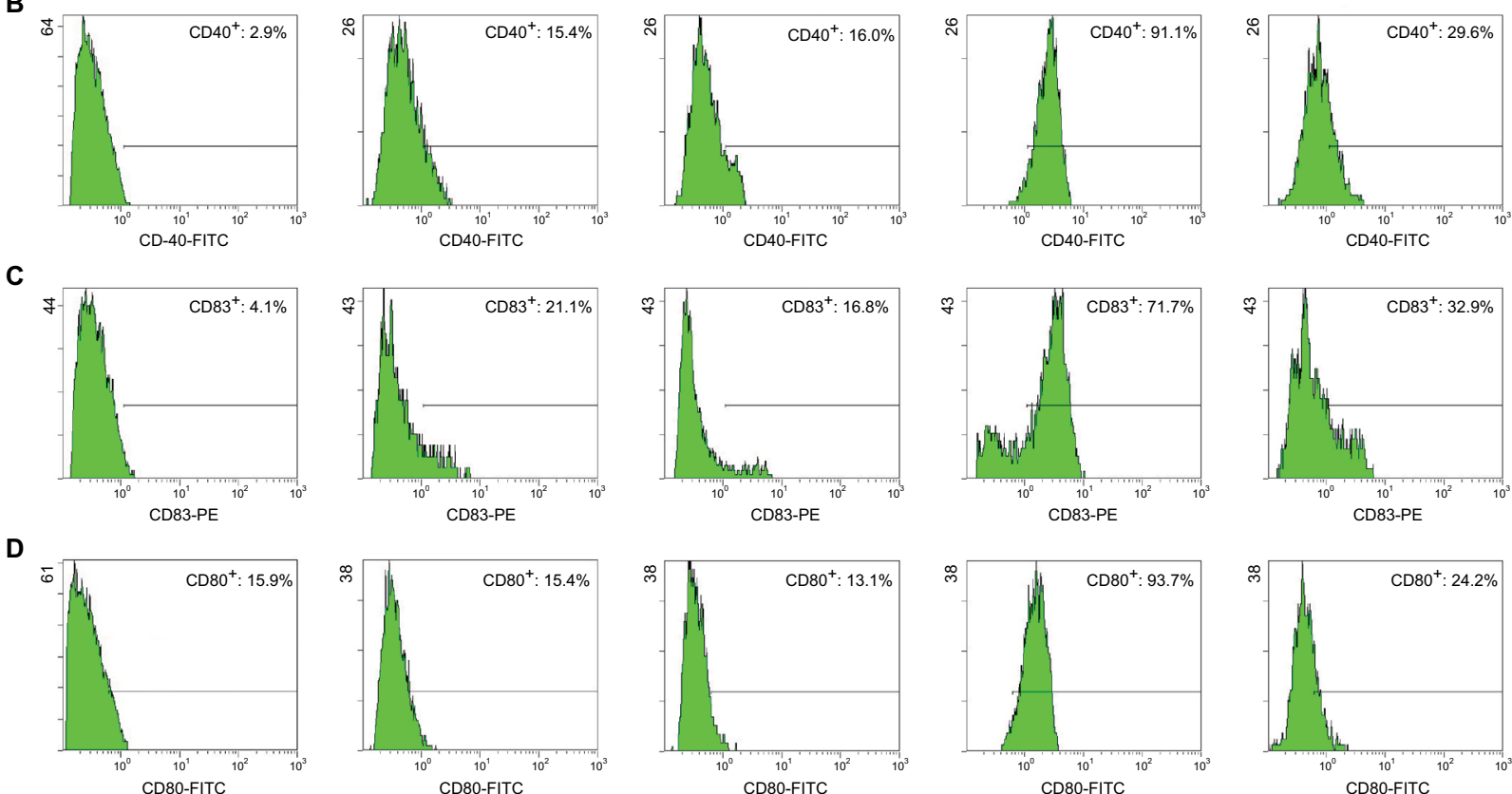

E
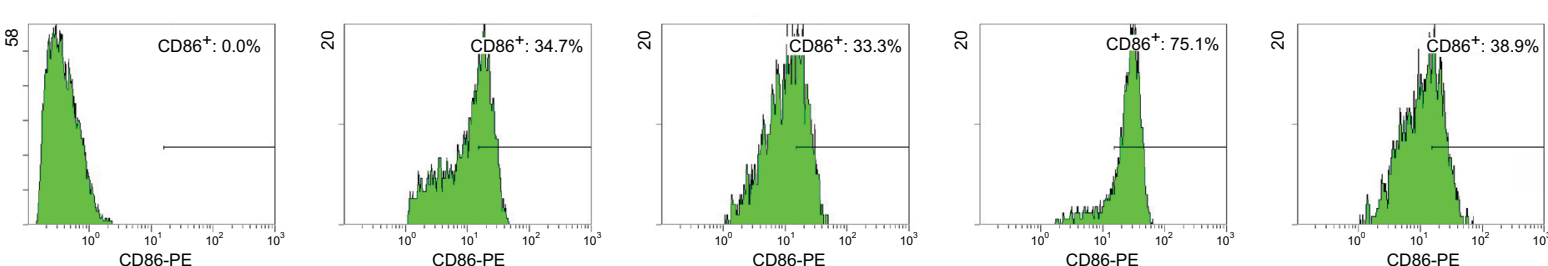

Figure 3 Flow cytometry histograms showing the expression of functional surface molecules of DC.

Notes: Groups of DC (imDC, imDC-AFP, mDC, and mDC-AFP) from one of the biological samples were treated with fluorescence-conjugated antibody against five functional surface molecules of DC (HLA-DR, CD40, CD83, CD80, and CD86) and read by flow cytometry. Percentage of cells with positive expression was calculated relative to the total number of DC read by flow cytometer. (A) Flow cytometry histogram of HLA-DR. (B) CD40. (C) CD83. (D) CD80. (E) CD86. Gating was determined based on the fluorescence values of cells in $\mathrm{mDC}$ group, in which the respective surface molecules are at their expected normal expression level after LPS maturation. Cells with a value below the threshold were considered to suboptimally express each respective surface molecule and was denoted in each dot plot as negative cells. Positive cell percentage shown in each dot plot was calculated relative to the total number of DC read by the flow cytometer.

Abbreviations: DC, dendritic cell; imDC, immature DC; imDC-AFP, alpha-fetoprotein-treated imDC; mDC, mature DC; mDC-AFP, alpha-fetoprotein-treated mDC; LPS, lipopolysaccharide.

results proving that AFP similarly induces tolerant and immunosuppressive responses from a wide range of immune cells including $\mathrm{T}$ cells, ${ }^{5}$ the result obtained in this study strictly demonstrated the effect of AFP through DC, instead of any AFP effect directly toward $\mathrm{CD} 4^{+} \mathrm{T}$ cells.

\section{Discussion}

AFP has previously been proven in many ways to be disrupting the functions of DC as an antigen-presenting cell. Starting from causing a decrease in inflammatory cytokines such as
IL-12, ${ }^{8}$ blocking NF- $\kappa B$ signaling, ${ }^{9}$ and lowering its potential in activating NK cells ${ }^{7}$ and T cells. ${ }^{12}$ Rather than causing many specific singular changes, AFP causes a certain dysfunction in its overall function as an immune system activator. ${ }^{4-6}$ This dysfunction shares a close similarity to the characteristics of tolerogenic DC, which is the state of DC when faced against self-antigens or harmless antigens, ${ }^{15}$ causing it to suppress the immune reaction against those antigens and tolerate their presence in our body. Instead of only lowering DC potential as an APC, AFP also turned DC to suppress the functions of 
Table 2 Percentage of DC with positive expression of functional surface molecules

\begin{tabular}{|c|c|c|c|c|c|c|}
\hline \multirow{2}{*}{$\begin{array}{l}\text { Surface } \\
\text { molecule }\end{array}$} & \multirow[t]{2}{*}{ Group } & \multicolumn{5}{|c|}{ Cells with positive molecule expression (\%) } \\
\hline & & \#I & \#2 & \#3 & Average & SD \\
\hline \multirow[t]{6}{*}{ HLA-DR } & $\operatorname{imDC}$ & $64.2 \%$ & $55.8 \%$ & $58.3 \%$ & $59.4 \%$ & $4.3 \%$ \\
\hline & imDC-AFP & $62.9 \%$ & $62.3 \%$ & $71.5 \%$ & $65.6 \%$ & $5.1 \%$ \\
\hline & $\mathrm{mDC}$ & $90.2 \%$ & $88.2 \%$ & $94.3 \%$ & $90.9 \%$ & $3.1 \%$ \\
\hline & mDC-AFP & $71.8 \%$ & $76.1 \%$ & $71.7 \%$ & $73.2 \%$ & $2.5 \%$ \\
\hline & $p$-value & \multicolumn{2}{|c|}{ imDC vs mDC-AFP } & & \multicolumn{2}{|l|}{0.0643} \\
\hline & & \multicolumn{2}{|c|}{ mDC vs mDC-AFP } & & \multicolumn{2}{|l|}{$0.0285^{*}$} \\
\hline \multirow[t]{6}{*}{ CD40 } & imDC & $15.4 \%$ & $24.3 \%$ & $17.9 \%$ & $19.2 \%$ & $4.6 \%$ \\
\hline & imDC-AFP & $16.0 \%$ & $10.4 \%$ & $21.5 \%$ & $16.0 \%$ & $5.6 \%$ \\
\hline & $\mathrm{mDC}$ & $91.1 \%$ & $85.2 \%$ & $82.2 \%$ & $86.2 \%$ & $4.5 \%$ \\
\hline & mDC-AFP & $29.6 \%$ & $34.9 \%$ & $35.0 \%$ & $33.2 \%$ & $3.1 \%$ \\
\hline & $p$-value & \multicolumn{2}{|c|}{ imDC vs mDC-AFP } & & \multicolumn{2}{|l|}{$0.0176 *$} \\
\hline & & \multicolumn{2}{|c|}{$\mathrm{mDC}$ vs mDC-AFP } & & \multicolumn{2}{|l|}{$0.0066 *$} \\
\hline \multirow[t]{6}{*}{ CD83 } & imDC & $21.1 \%$ & $12.4 \%$ & $16.5 \%$ & $16.7 \%$ & $4.4 \%$ \\
\hline & imDC-AFP & $16.8 \%$ & $23.1 \%$ & $12.9 \%$ & $17.6 \%$ & $5.1 \%$ \\
\hline & $\mathrm{mDC}$ & $71.7 \%$ & $75.6 \%$ & $72.6 \%$ & $73.3 \%$ & $2.0 \%$ \\
\hline & $\mathrm{mDC}-\mathrm{AFP}$ & $32.9 \%$ & $36.7 \%$ & $38.9 \%$ & $36.2 \%$ & $3.0 \%$ \\
\hline & $p$-value & \multicolumn{2}{|c|}{ imDC vs mDC-AFP } & & \multicolumn{2}{|l|}{$0.0375^{*}$} \\
\hline & & \multicolumn{2}{|c|}{ mDC vs mDC-AFP } & & \multicolumn{2}{|l|}{$0.0021 *$} \\
\hline \multirow[t]{6}{*}{ CD80 } & imDC & $15.4 \%$ & $21.7 \%$ & $14.5 \%$ & $17.2 \%$ & $3.9 \%$ \\
\hline & imDC-AFP & $13.1 \%$ & $11.1 \%$ & $10.2 \%$ & $11.5 \%$ & $1.5 \%$ \\
\hline & $\mathrm{mDC}$ & $93.7 \%$ & $93.7 \%$ & $91.6 \%$ & $93.0 \%$ & $1.2 \%$ \\
\hline & mDC-AFP & $24.2 \%$ & $17.3 \%$ & $22.2 \%$ & $21.2 \%$ & $3.6 \%$ \\
\hline & $p$-value & \multicolumn{2}{|c|}{ imDC vs mDC-AFP } & & \multicolumn{2}{|l|}{0.4408} \\
\hline & & \multicolumn{2}{|c|}{ mDC vs mDC-AFP } & & \multicolumn{2}{|l|}{$0.0010 *$} \\
\hline \multirow[t]{6}{*}{ CD86 } & $\operatorname{imDC}$ & $34.7 \%$ & $35.2 \%$ & $29.0 \%$ & $33.0 \%$ & $3.4 \%$ \\
\hline & imDC-AFP & $33.3 \%$ & $36.1 \%$ & $40.9 \%$ & $36.8 \%$ & $3.8 \%$ \\
\hline & $\mathrm{mDC}$ & $75.1 \%$ & $70.2 \%$ & $68.2 \%$ & $71.2 \%$ & $3.6 \%$ \\
\hline & mDC-AFP & $38.9 \%$ & $37.7 \%$ & $37.0 \%$ & $37.9 \%$ & $1.0 \%$ \\
\hline & $p$-value & \multicolumn{2}{|c|}{ imDC vs mDC-AFP } & & \multicolumn{2}{|l|}{0.0947} \\
\hline & & \multicolumn{2}{|c|}{ mDC vs mDC-AFP } & & \multicolumn{2}{|l|}{$0.0020^{*}$} \\
\hline
\end{tabular}

Notes: The table shows the percentage values of sampled DC with positive expression as depicted in Figure 3 histograms (sample \#I) and two additional technical replicates from the same biological blood donor (\#2 and \#3; histogram not shown). ${ }^{*} p<0.05$.

Abbreviations: DC, dendritic cell; imDC, immature DC; imDC-AFP, alpha-fetoprotein-treated imDC; mDC, mature DC; mDC-AFP, alpha-fetoprotein-treated mDC.

Table 3 Quantified TGF- $\beta$ concentration detected in the culture medium of DC

\begin{tabular}{llllll}
\hline Group & \multicolumn{5}{l}{ TGF- $\beta$ concentration $(\mathbf{p g} / \mathbf{m L})$} \\
\cline { 2 - 6 } & $\# \mathbf{1}$ & $\mathbf{\# 2}$ & $\mathbf{\# 3}$ & Average & SD \\
\hline imDC & 5.4 & 0.1 & 0.0 & 1.8 & 3.1 \\
imDC-AFP & 47.4 & 23.1 & 19.5 & 30.0 & 15.2 \\
mDC & 53.3 & 21.2 & 38.3 & 37.6 & 16.1 \\
mDC-AFP & 58.7 & 77.5 & 78.4 & 71.5 & 11.1 \\
\hline p-value & imDC vs imDC-AFP & $0.034511^{*}$ & \\
& $m D C$ vs mDC-AFP & $0.039614^{*}$ & \\
\hline
\end{tabular}

Notes: The table shows the concentration values of TGF- $\beta$ in the culture medium used to culture DC from four different assigned treatment groups (imDC, imDCAFP, $\mathrm{mDC}$, and $\mathrm{mDC}-\mathrm{AFP}$ ) starting from the first day. The results were obtained from three biological replicates. $* p<0.05$.

Abbreviations: DC, dendritic cell; imDC, immature DC; imDC-AFP, alphafetoprotein-treated imDC; mDC, mature DC; mDC-AFP, alpha-fetoprotein-treated $\mathrm{mDC}$

other immune cells, which widely involves the generation and participation of regulatory T cells. ${ }^{12,15-17}$ Recently, culturing $\mathrm{DC}$ in AFP containing medium was proven to cause DC to stimulate significantly less $\mathrm{CD} 4^{+}$and $\mathrm{CD} 8^{+} \mathrm{T}$ cells. ${ }^{12}$ One of the aims of this study is to trace the root cause upstream of research-proven DC dysfunctions and then confirm our finding by reproducing the same effect. In this study, we traced the root cause of the lowered T-cell proliferation stimulating potential discovered by Pardee et al.12 We also expected it to be closely related to the downregulated expression of DC maturation surface markers.

First, our result showed a significant reduction of LPS on imDC-AFP surface compared to normal untreated imDC. Both groups were significantly different $(p<0.05)$ (Table 1$)$. AFP might had been bound onto the LPS binding site, eliminating its ability to bind onto LPS receptor on DC surface and therefore neutralizing the maturation signal in the culture and cancelling the maturation of DC to a certain degree. ${ }^{18}$ Another high possibility is that AFP, being a tumor-derived factor, promoted DC transition into the antigen-tolerant tolerogenic DC during its 6 days in MDDC culture. One of the characteristics of tolerogenic $\mathrm{DC}$ is being less responsive to antigenic activation signal. LPS 
A

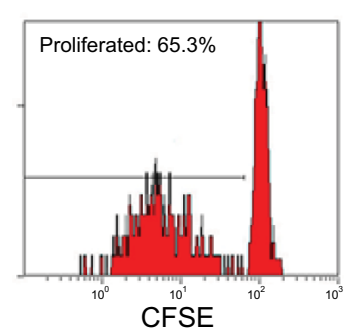

B

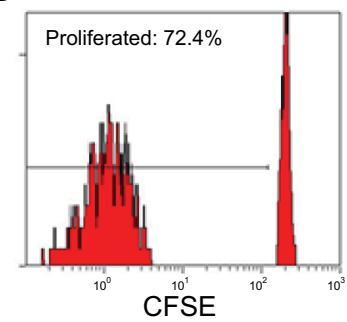

C

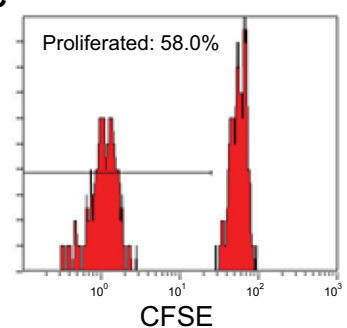

imDC-AFP
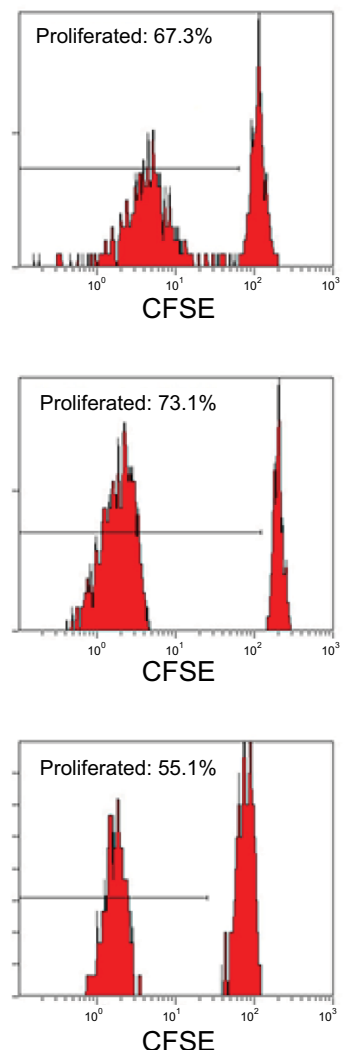

$\mathrm{mDC}$
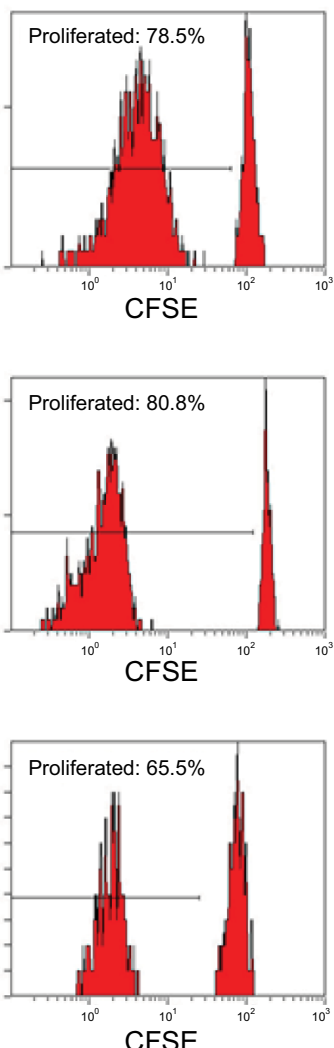

mDC-AFP
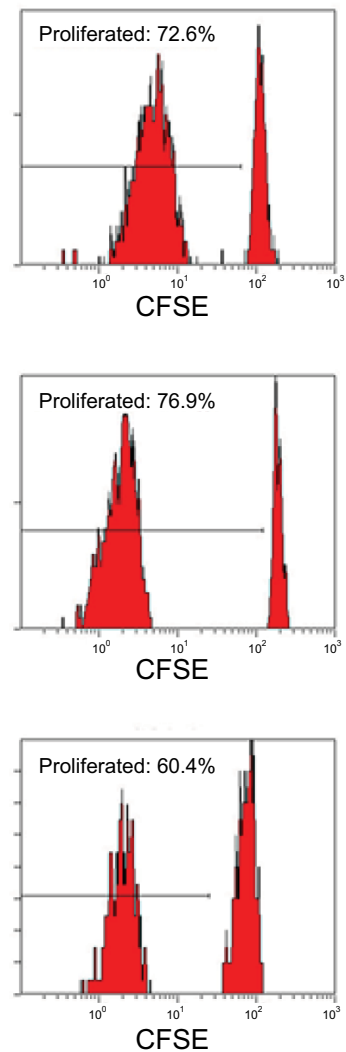

Figure 4 Flow cytometry dot plots and histograms showing the proliferation of DC-induced CD4+ T cells.

Notes: Three batches of PBMC sample were treated with CFSE prior to 5 days in a ratio of $1: 10$ in MLR culture with DC from four assigned treatment groups (imDC, imDC-AFP, mDC, and mDC-AFP). MLR culture was followed by flow cytometry run. Gating was determined based on the fluorescence values of unproliferated CFSE-labeled cells, where intracellular CFSE was still at the maximum level. Cells with values below threshold were considered as proliferated cells and had their percentage calculated relative to the total number of PBMC read by the flow cytometer. PHA was used in this experiment as a cell proliferation positive control. (A-C) CD4+ gated histograms of CFSE-labeled imDC, imDC-AFP, mDC, and mDC-AFP treatment group-stimulated PBMC from the three biological replicates.

Abbreviations: DC, dendritic cell; PBMC, peripheral blood mononuclear cell; imDC, immature DC; imDC-AFP, alpha-fetoprotein-treated imDC; mDC, mature DC; mDCAFP, alpha-fetoprotein-treated mDC; CFSE, carboxyfluorescein succinimidyl ester; MLR, mixed lymphocyte reaction.

Table 4 Percentage of proliferated CD4+ T cells after different DC stimulations

\begin{tabular}{|c|c|c|c|c|c|c|c|c|c|c|c|c|c|}
\hline \multirow[t]{2}{*}{ Group } & \multicolumn{3}{|c|}{$\begin{array}{l}\text { Proliferated (\%) } \\
\text { Raw values }\end{array}$} & \multicolumn{5}{|c|}{$\begin{array}{l}\text { Proliferated }(\%) \\
\text { imDC (\%) Proliferated } \times 100 \%\end{array}$} & \multicolumn{5}{|c|}{$\begin{array}{l}\text { Proliferated }(\%) / \\
\text { mDC (\%) Proliferated } \times 100 \%\end{array}$} \\
\hline & $\# I$ & $\# 2$ & \#3 & $\# 1$ & $\# 2$ & $\# 3$ & Average & SD & $\#$ \# & $\# 2$ & \#3 & Average & SD \\
\hline imDC & $65.3 \%$ & $72.4 \%$ & $58.0 \%$ & $100.0 \%$ & $100.0 \%$ & $100.0 \%$ & $100.0 \%$ & - & & & & & \\
\hline imDC-AFP & $67.3 \%$ & $73.1 \%$ & $55.1 \%$ & $103.1 \%$ & $101.0 \%$ & $95.0 \%$ & $99.7 \%$ & $4.2 \%$ & & & & & \\
\hline $\mathrm{mDC}$ & $78.5 \%$ & $80.8 \%$ & $65.5 \%$ & $120.2 \%$ & $111.6 \%$ & $112.9 \%$ & $114.9 \%$ & $4.6 \%$ & $100.0 \%$ & $100.0 \%$ & $100.0 \%$ & $100.0 \%$ & - \\
\hline mDC-AFP & $72.6 \%$ & $76.9 \%$ & $60.4 \%$ & $111.2 \%$ & $106.2 \%$ & $104.1 \%$ & $107.2 \%$ & $3.6 \%$ & $92.5 \%$ & $95.2 \%$ & $92.2 \%$ & $93.3 \%$ & $1.6 \%$ \\
\hline$p$-value & & & & $\operatorname{imDC} v$ & $\mathrm{mDC}-\mathrm{Al}$ & & $0.0264^{*}$ & & $\mathrm{mDC}$ vs & mDC-AFF & & $0.0021 *$ & \\
\hline
\end{tabular}

Notes: Left column: raw percentage values of proliferated CD4+ T cells taken directly from the histogram. Middle column: proliferated CD4+ T cells' raw values from the left column, normalized with imDC group taken as the control, while the treated groups were compared relative to imDC in percentage. Right column: proliferated CD4+ $T$ cells' raw values from the left column, normalized with non-treated $\mathrm{mDC}$ group taken as the control, while mDC-AFP was compared relative to non-treated mDC in percentage, done particularly to show similar proliferation decrease due to DC AFP prior treatment in all three biological replicates. * $p<0.05$.

Abbreviations: DC, dendritic cell; imDC, immature DC; imDC-AFP, alpha-fetoprotein-treated imDC; mDC, mature DC; mDC-AFP, alpha-fetoprotein-treated mDC.

receptors (TLR4) on DC surface might have been compromised, whether had its structure altered or possibly had its expression suppressed due to AFP exposure. Decreased functional LPS receptor could lower the maturation signal magnitude overall, which leads to generation of abnormal immunogenic $\mathrm{mDC}$ and inherent degeneration of its function as an APC.
The expressions of HLA-DR, CD40, CD80, CD83, and CD86 were downregulated on $\mathrm{mDC}$-AFP surface compared to normal untreated $\mathrm{mDC}$. We can see significant decreases in the expression of $\mathrm{mDC}$ surface molecules (class II MHC, costimulatory, and costimulatory promoter molecules) being highly responsible for the activation of proliferation and differentiation 
of immune cells, particularly T cells. AFP does not inflict any apparent downregulation of expression on imDC. However, the inhibition is clear on $\mathrm{mDC}$. Rather than decreasing the expression, it is more logical to say that AFP simply prevented the upregulation of these molecules during the maturation process, making it a less potent proliferation activator downstream the immunogenic effector immune response mechanism.

AFP caused a significant increase in the secretion of anti-inflammatory cytokine TGF- $\beta$ by DC. It was also noteworthy that AFP also altered TGF- $\beta$ secretion even when DC was still in its immature state, instead of only affecting the mDC. mDC increased the secretion of TGF- $\beta$ in order to maintain the immunogenicity balance and prevent the effector cells from getting overstimulated and causing selfdamage. ${ }^{10}$ Judging from these data, AFP was able to induce the transition of DC into being tolerogenic regardless of its maturity. Immunoregulatory effects of AFP, besides reducing the effector-type immune response, also increase the regulatory-type immune response. This immunoregulatory effect begins at characteristic and functional transition of monocyte into a tolerogenic immature DC, which was caused by exposure to AFP since monocyte. While there is no direct reference yet on the mechanism of AFP in affecting TGF- $\beta$ pathway, tolerogenic DC is known to secrete a higher level of TGF- $\beta$ than normal DC, regardless of its maturity state. ${ }^{15}$ Tolerogenic DC cannot respond toward maturation stimulation normally, making it unable to be fully matured and reduce its potential as an APC. ${ }^{15}$ The transition of imDC toward becoming tolerogenic possibly lowers down the expression of TLR4 on its surface. This lowers the maturation induction by its agonist, bacterial LPS, and thus DC responses toward foreign antigens.

A study done by Pardee et al found out that AFP-treated DC decreased its ability to stimulate the proliferation of T cells from both subsets of $\mathrm{CD}^{+}$and $\mathrm{CD}^{+} .{ }^{12}$ This finding points out that regardless of the type of immune response generated, the strength of the generated response itself would also have been compromised by AFP exposure. Also in accordance with the same study by Pardee et al, our results showed that one of the immunoregulatory effects of AFP is disruption of the maturation of DC. AFP disrupted the ability of $\mathrm{mDC}$ to induce proliferation on $\mathrm{CD}^{+} \mathrm{T}$-cell population in a similar trend as its effects on functional surface molecules on DC surface: AFP treatment decreased $\mathrm{mDC}$ potential in stimulating the proliferation of $\mathrm{CD}^{+} \mathrm{T}$ cells into a level slightly higher than it was in imDC. Less $\mathrm{CD}^{+} \mathrm{T}$ cells simply means that less immune system signal messengers are to be expected when foreign antigen threats are present, which could in turn lead to less generation of every other cell involved in effector immune response, and results in a weakened, less hostile, and more lenient response against the antigen.

From the trend of the results, AFP effects on LPS binding onto DC surface, DC functional surface molecules, and DC-induced $\mathrm{CD}^{+}{ }^{+} \mathrm{T}$-cell proliferation are parts of a chain reaction. Decreased imDC interaction with its maturation signal LPS inhibits the upregulation of functional surface molecule expression, causing mDC-AFP to express much less functional surface molecules compared to normal $\mathrm{mDC}$. These functional surface molecules are responsible for the clonal expansion of T cells; this expression reduction results in less proliferation of $\mathrm{CD}^{+} \mathrm{T}$ cell, a major activator of many effector immune cell differentiation and clonal expansion. Considering the fact that $\mathrm{CD}^{+} \mathrm{T}$ cells are the key activator of almost all kinds of cellular adaptive immune response, these three effects of AFP on DC surely lead to the weakening of overall effector immune response.

Exposure of DC toward AFP during its differentiation from monocytes decreased the amount of expressed TLR4 on its immature-state cell surface (Figure S3). Having subnormal TLR4 expression level at the maturation signaling front end, this should have a detrimental effect on the resulting DC maturity. Thus, even without contact inhibition, such as active site blockade and molecule sequestration on LPS and TLR4 interaction, AFP is already able to inflict its immunosuppressive effects on DC.

Such contact inhibition events are still logically possible and are left to be investigated further due to the limitation of our experimental design. There is a possibility that AFP binds onto certain nonspecific receptors that it structurally prevents the contact and binding of LPS molecule onto TLR4, particularly if it binds onto and blocks the TLR4 molecule active site itself. Another possibility is free LPS molecule sequestration by free AFP molecules in the medium during the 2 days of LPS stimulation, which prevents LPS from any interaction with TLR4. These events could contribute to the strength of AFP immunosuppressive effects on DC. Nevertheless, this confirms the existence of TLR4 modulating effect of AFP on DC, and some of its functions as an APC downstream the adaptive immunity activation.

Although the flow of AFP disruptions toward DC functions starting from the weakened maturation signal down to the reduced proliferation-stimulating potential is very logical, this conclusion is still not entirely irrefutable. There are several limitations in this study, making some probabilities of AFP mechanisms of disruption being left uninvestigated. One such probability that we would like to point out is the 
probability of AFP's direct effect toward $\mathrm{CD}^{+}$proliferation. While this direct effect is already proven to be true, ${ }^{5}$ more experiments are needed to safely claim that AFP affects $\mathrm{CD}^{+} \mathrm{T}$ cells solely through inhibition of DC maturation. Another probability is that AFP molecule somehow interferes with the proliferation stimulation signaling between $\mathrm{DC}$ and $\mathrm{CD}^{+} \mathrm{T}$ cells, just by being present on the spot during the stimulation of $\mathrm{CD}^{+} \mathrm{T}$ cells, without bringing about any of its immunosuppressive effects on the DC beforehand. This is somewhat possible particularly due to the first and second T-cell activation signals, which are known to require direct contact between surface molecules of both cells.

\section{Acknowledgments}

This study was fully funded by Mochtar Riady Institute for Nanotechnology. The authors thank Akterono Dwi Budiyati, M. Biomed, for experimental counsel and Dr. rer. nat. Ivet Suriapranata, Dr. Juandy Jo, and Atul Philip Varghese, M Eng, for critical discussions.

\section{Disclosure}

The authors report no conflicts of interest in this work.

\section{References}

1. Zhou L, Liu J, Luo F. Serum tumor markers for detection of hepatocellular carcinoma. World J Gastroenterol. 2006;12(8):1175-1181.

2. Arrieta O, Cacho B, Morales-Espinosa D, Ruelas-Villavicencio A, Flores-Estrada D, Hernández-Pedro N. The progressive elevation of alpha fetoprotein for the diagnosis of hepatocellular carcinoma in patients with liver cirrhosis. BMC Cancer. 2007;7:28.

3. Tangkijvanich P, Anukulkarnkusol N, Suwangool P, et al. Clinical characteristics and prognosis of hepatocellular carcinoma: analysis based on serum alpha-fetoprotein levels. J Clin Gastroenterol. 2000;31(4):302-308.
4. Ninomiya T, Akbar SM, Masumoto T, Horiike N, Onji M. Dendritic cells with immature phenotype and defective function in the peripheral blood from patients with hepatocellular carcinoma. $J$ Hepatol. 1999;31(2):323-331.

5. Um SH, Mulhall C, Alisa A, et al. Alpha-fetoprotein impairs APC function and induces their apoptosis. J Immunol. 2004;173(3):1772-1778.

6. Pinzon-Charry A, Maxwell T, López JA. Dendritic cell dysfunction in cancer: a mechanism for immunosuppression. Immunol Cell Biol. 2005;83(5):451-461.

7. Yamamoto M, Tatsumi T, Miyagi T, et al. $\alpha$-Fetoprotein impairs activation of natural killer cells by inhibiting the function of dendritic cells. Clin Exp Immunol. 2011;165(2):211-219.

8. Setiyono A, Budiyati AD, Purwantomo S, et al. Immunoregulatory effects of AFP domains on monocyte-derived dendritic cell function. BMC Immunol. 2011;12:4.

9. Budiyati AD, Setiyono A, Tarigan E, Wibowo H. The effect of alpha fetoprotein on NF- $\kappa B$ translocation in lipopolysaccharide induced monocyte-derived dendritic cell. Med J Indones. 2012;21(2):97-101.

10. Abbas AK, Lichtman AH, Pillai S. Cellular and Molecular Immunology. 6th ed. Philadelphia, PA: Saunders Elsevier; 2010.

11. Lehar SM, Bevan MJ. Immunology: polarizing a T-cell response. Nature. 2004;430(6996):150-151.

12. Pardee AD, Shi J, Butterfield LH. Tumor-derived $\alpha$-fetoprotein impairs the differentiation and $\mathrm{T}$ cell stimulatory activity of human dendritic cells. J Immunol. 2014;193(11):5723-5732.

13. Alisa A, Boswell S, Pathan AA, Ayaru L, Williams R, Behboudi S. Human CD4(+) T cells recognize an epitope within alpha-fetoprotein sequence and develop into TGF-beta-producing CD4(+) T cells. $J$ Immunol. 2008;180(7):5109-5117.

14. Janikashvili N, Bonnotte B, Katsanis E, Larmonier N. The dendritic cell-regulatory $\mathrm{T}$ lymphocyte crosstalk contributes to tumor-induced tolerance. Clin Dev Immunol. 2011;2011:430394.

15. Torres-Aguilar H, Blank M, Jara LJ, Shoenfeld Y. Tolerogenic dendritic cells in autoimmune diseases: crucial players in induction and prevention of autoimmunity. Autoimmun Rev. 2010;10(1):8-17.

16. Maldonado RA, von Andrian UH. How tolerogenic dendritic cells induce regulatory T cells. Adv Immunol. 2010;108:111-165.

17. Yamazaki S, Inaba K, Tarbell KV, Steinman RM. Dendritic cells expand antigen-specific Foxp3+CD25+CD4+ regulatory T cells including suppressors of alloreactivity. Immunol Rev. 2006;212:314-329.

18. Rosenfeld Y, Papo N, Shai Y. Endotoxin (lipopolysaccharide) neutralization by innate immunity host-defense peptides. Peptide properties and plausible modes of action. J Biol Chem. 2006;281(3):1636-1643. 


\section{Supplementary materials}

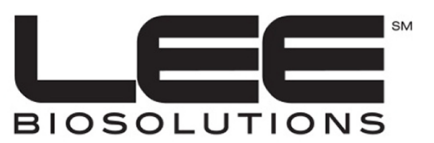

2924 MARY AVENUE, ST. LOUIS, MISSOURI, USA 63144 T: 314.968 .1091 F: 314.968 .9851 WWW.LEEBIO.COM

\section{SPECIFICATION}

\section{Alpha Fetoprotein}

Human Cord Serum

\section{GENERAL INFORMATION}

\begin{tabular}{llll}
\hline Catalogue Number & $105-11$ & Storage Temperature & $2-8^{\circ} \mathrm{C}$ \\
Lot Number & Reported & Storage Note & Do Not Freeze \\
Synonyms & AFP, alpha-1-fetoprotein, & Shipping Note & Cold packs \\
& alpha fetal protein & Received By & - \\
& & Receipt Date & - \\
\hline
\end{tabular}

\section{PRODUCT INFORMATION}

\begin{tabular}{ll}
\hline Source & Human Cord Serum \\
Form & 0.2 micron filtered solution in tris buffered saline, $\mathrm{pH} 7.5$ with $0.1 \%$ sodium azide. \\
Appearance & Clear, colorless to straw liquid. \\
Protein & $1.5-2.0 \mathrm{mg} / \mathrm{mL}\left(\mathrm{E}^{0.1 \%}{ }_{280 \mathrm{~nm}}=0.52\right)$ \\
Purity & $\geq 99 \%(\mathrm{SDS}-\mathrm{PAGE}$, Western $)$ \\
Identity & Confirmed by Western Blot and ELISA \\
Recertification & Three years from manufacture date.
\end{tabular}

\section{HANDLING INFORMATION}

\begin{tabular}{ll}
\hline Handling & Handle as a potentially hazardous substance. Refer to MSDS on website. \\
Recovery & If small volume, centrifuge briefly to assure complete recovery,
\end{tabular}

\section{CERTIFIED BY:}

DATE:

Non-Infectious Disease Statement: Non-reactive for HIV-1/HCV/ HBV by NAT, HBsAg, HCV Ab, HIV $1 \& 2$ Ab, and RPR by currently approved FDA methods. However, because no test method can offer complete assurance that infectious agents are absent, this material should be handled at the Biosafety Level 2 (BSL 2) as recommended for any potentially infectious human serum or blood specimen in the CDC/NIH manual "Bio-safety in microbiological and Biomedical Laboratories", 1999. This material is sold for in-vitro diagnostic use only in manufacturing and research. This material is not suitable for human use. We certify this to be true to the best of our knowledge. The statements herein are offered for informational purposes only and are intended to be used solely for your consideration, investigation and verification only. It is up to the user to undertake sufficient verification and testing to determine the suitability for their own particular purpose. Use of product determined by end user.

Figure SI Human cord blood serum AFP technical datasheet.

Note: Detailed specifications of the commercially available cord blood normal healthy adult human-obtained AFP, purchased and used in this study, showing the purity number of $\geq 99 \%$ according to SDS-PAGE and western blot tests.

Abbreviation: AFP, alpha-fetoprotein. 


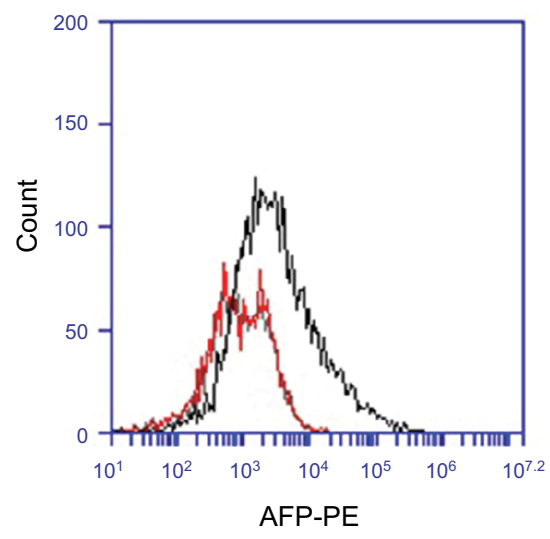

Unlabeled

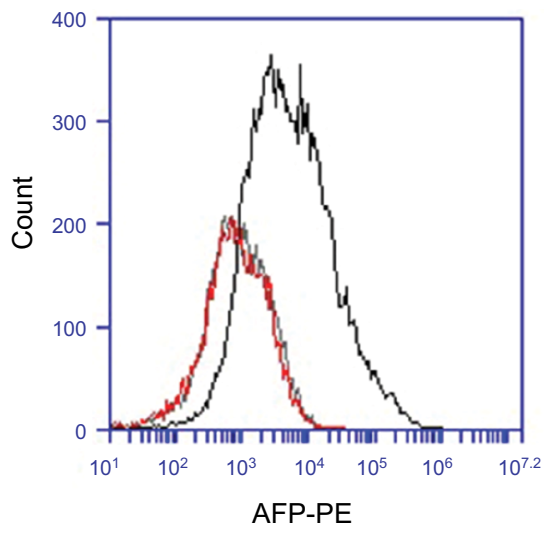

imDC-AFP

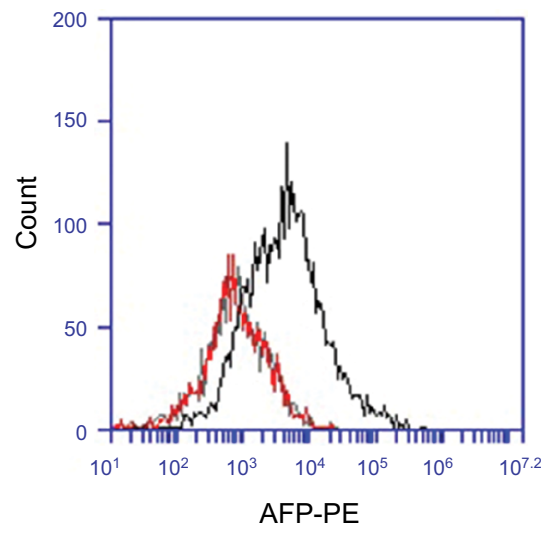

$\operatorname{imDC}$

Figure S2 Flow cytometry histogram showing the fluorescence intensity of anti-AFP-PE on the surface of DC.

Notes: The results were obtained through three technical replicates from a single biological blood sample. The fluorescence intensity at PE light-level wavelength reflected the number of anti-AFP successfully bound onto DC surface and consequently the number of successfully bound AFP molecules on DC surface.

Abbreviations: AFP, alpha-fetoprotein; DC, dendritic cell; imDC-AFP, AFP-treated immature DC.

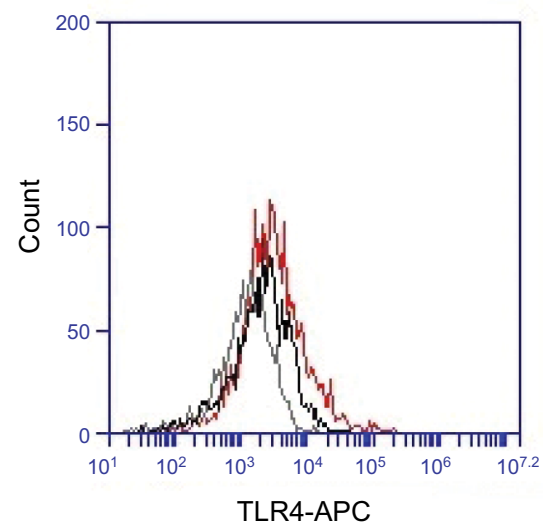

Unlabeled

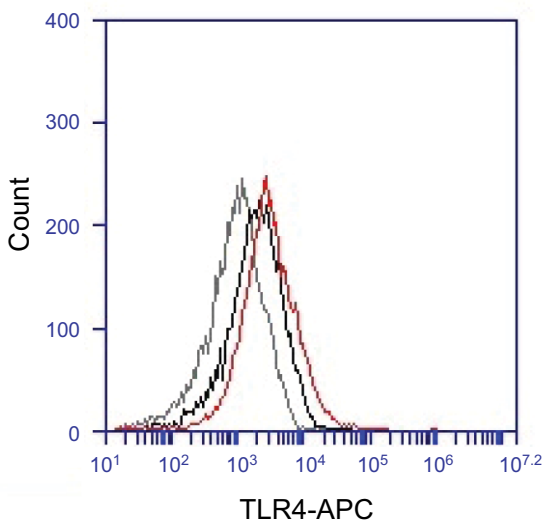

imDC-AFP

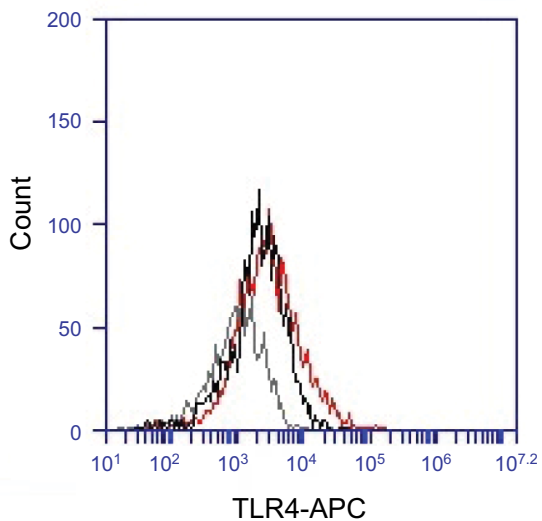

$\operatorname{imDC}$

Figure S3 Flow cytometry histogram showing the fluorescence intensity of anti-TLR4-APC on the surface of DC.

Notes: The results were obtained through three technical replicates from a single biological blood sample. The fluorescence intensity at APC light level wavelength reflected the number of anti-TLR4 successfully bound onto DC surface and consequently the number of available TLR4 molecules on DC surface.

Abbreviations: DC, dendritic cell; AFP, alpha-fetoprotein.

Table SI MFI of anti-AFP-PE labeled DC

\begin{tabular}{llllll}
\hline Group & MFI (AU) & & & & Average \\
\cline { 2 - 6 } & \#I & \#2 & \#3 & SD \\
\hline Unlabeled & $1,321.63$ & $1,449.30$ & $1,311.39$ & $1,360.77$ & 76.84 \\
imDC & $1,319.29$ & $1,323.18$ & $1,291.60$ & $1,311.36$ & 17.22 \\
imDC-AFP & $9,315.64$ & $12,122.92$ & $10,843.16$ & $10,760.57$ & 1405.46 \\
\hline p-value & imDC vs imDC-AFP & & & 0.003642 & \\
\hline
\end{tabular}

Notes: The table shows the MFI values of mean fluorescence intensity from the three technical replicates. $p$-value between imDC and imDC-AFP groups was calculated using two-tailed paired Student's $t$-test to observe and confirm the binding persistence of AFP molecules onto DC surface after repeated cell washing with PBS.

Abbreviations: MFI, mean fluorescence intensity; AFP, alpha-fetoprotein; DC, dendritic cell; imDC, immature DC; imDC-AFP, AFP-treated imDC. 
Table S2 MFI of anti-TLR4-APC labeled DC

\begin{tabular}{|c|c|c|c|c|c|}
\hline \multirow[t]{2}{*}{ Group } & \multicolumn{5}{|c|}{ MFI (AU) } \\
\hline & \#I & $\# 2$ & \#3 & Average & SD \\
\hline Unlabeled & $\mathrm{I}, 449.70$ & $1,312.02$ & $1,304.60$ & $\mathrm{I}, 355.44$ & 81.72 \\
\hline imDC & $5,311.53$ & $5,157.77$ & $5,195.45$ & $5,221.58$ & 80.14 \\
\hline imDC-AFP & $2,113.73$ & $2,534.07$ & $2,499.04$ & $2,382.28$ & 233.23 \\
\hline$p$-value & \multicolumn{3}{|c|}{ imDC vs imDC-AFP } & 0.004016 & \\
\hline
\end{tabular}

Notes: The table shows the MFI values of mean fluorescence intensity from the three technical replicates. $p$-value between imDC and imDC-AFP groups was calculated using two-tailed paired Student's $t$-test to see the effect of 6 days of AFP treatment on the number of available TLR4 molecules on DC surface.

Abbreviations: MFI, mean fluorescence intensity; DC, dendritic cell; imDC, immature DC; imDC-AFP, alpha-fetoprotein-treated imDC.

The Journal of Hepatocellular Carcinoma is an international, peerreviewed, open access journal that offers a platform for the dissemination and study of clinical, translational and basic research findings in this rapidly developing field. Development in areas including, but not limited to, epidemiology, vaccination, hepatitis therapy, pathology and molecular tumor classification and prognostication are all considered for publication. The manuscript management system is completely online and includes a very quick and fair peer-review system, which is all easy to use. Visit http://www.dovepress.com/testimonials.php to read real quotes from published authors.

Submit your manuscript here: https://www.dovepress.com/journal-of-hepatocellular-carcinoma-journal 